\title{
用于高性能超级电容器电极的栓皮栋基多孔活性炭的制备
}

\author{
许伟佳, 邱大平, 刘诗强, 李 敏, 杨儒 \\ (北京化工大学 材料电化学过程与技术北京市重点实验室, 化工资源有效利用国家重点实验室, 北京 100029)
}

摘 要: 本研究以空腔细胞组成的栓皮栋为原料, $\mathrm{KOH}$ 为活化剂制备了具有多孔结构的栓皮栎软木基多孔活性炭。 以此方法制得的活性炭呈薄片状外形, 最大比表面积达到 $2312 \mathrm{~m}^{2} / \mathrm{g}$, 具有特殊的微孔一介孔结构。在呈碱性的 $\mathrm{KOH}$ 三电极体系中, $0.1 \mathrm{~A} / \mathrm{g}$ 电流密度时比电容达 $296 \mathrm{~F} / \mathrm{g}$; 两电极体系中, $5 \mathrm{~A} / \mathrm{g}$ 时的比电容达到 $201 \mathrm{~F} / \mathrm{g}$, 循环 5000 次后 电容保持率达 $99.5 \%$ 。在呈中性的 $\mathrm{Na}_{2} \mathrm{SO}_{4}$ 两电极体系中, 电流密度 $0.5 \mathrm{~A} / \mathrm{g}(174 \mathrm{~F} / \mathrm{g})$ 至 $50 \mathrm{~A} / \mathrm{g}(140 \mathrm{~F} / \mathrm{g})$ 时电容保持 率达 $80.5 \%$, 倍率性能良好, 能量密度高达 $19.62 \mathrm{Wh} / \mathrm{kg}$ 。

关 键 词: 软木; 活性炭; 互联孔结构; 超级电容器

中图分类号: O613 文献标识码: A

\section{Preparation of Cork-derived Porous Activated Carbon for High Performance Supercapacitors}

\begin{abstract}
XU Wei-Jia, QIU Da-Ping, LIU Shi-Qiang, LI Min, YANG Ru
(Beijing Key Laboratory of Electrochemical Process and Technology for Materials, The State Key Laboratory of Chemical Resource Engineering, Beijing University of Chemical Technology, Beijing 100029, China)
\end{abstract}

\begin{abstract}
The quercus variabilis cork made up of cavity cells is used as raw material. Herein, the cork-derived activated carbon with the various pores was successfully prepared by the facile carbonization of cork followed by chemical activation. The as-prepared activated carbon sheets possess large specific surface area $\left(2312 \mathrm{~m}^{2} / \mathrm{g}\right)$ and unique interconnected pores. As a result, it shows excellent electrochemical performance as electrode material for supercapacitors. In three electrode system of $\mathrm{KOH}$, it exhibits a high specific capacitance of $296 \mathrm{~F} / \mathrm{g}$ at a current density of $0.1 \mathrm{~A} / \mathrm{g}$. The assembled symmetric supercapacitor shows a high specific capacitance of $201 \mathrm{~F} / \mathrm{g}$ at $5 \mathrm{~A} / \mathrm{g}$, with a good cycling stability of $99.5 \%$ capacitance retention after 5000 cycles. In two electrode system of $\mathrm{Na}_{2} \mathrm{SO}_{4}$, the symmetric supercapacitor displays a good rate performance of $80.5 \%$ retention from $0.5 \mathrm{~A} / \mathrm{g}(174 \mathrm{~F} / \mathrm{g})$ to $50 \mathrm{~A} / \mathrm{g}$ $(140 \mathrm{~F} / \mathrm{g})$ and a high energy density of $19.62 \mathrm{Wh} / \mathrm{kg}$.
\end{abstract}

Key words: cork; activated carbon; interconnected pores; supercapacitor

生物质材料是一类广泛存在于自然界的优质可 再生材料, 结构精细、功能各异, 难以被人工合成材 料取代。这些结构各异的生物质材料不仅可以为人 类提供丰富的, 具有特殊结构的原料, 还可以为人 类开发设计具有精致结构、特殊功能的新材料提供 无限的灵感。近年来, 能源匮乏和环境污染等问题
日益严峻, 来源广泛、储量大、可再生、低污染的 生物质材料受到了越来越多的关注 ${ }^{[1-2]}$ 。

生物质活性炭是由各种富含炭元素的生物质材 料在惰性气氛保护下高温煅烧形成的具有特定结构 的炭材料。生物质活性炭不仅具有传统活性炭良好 的化学惰性、耐热性、高导电性、高导热性等物理

收稿日期：2018-09-13; 收到修改稿日期：2018-12-11

基金项目：国家自然科学基金(51372012, 51432003) National Natural Science Foundation of China $(51372012,51432003)$

作者简介：许伟佳(1990-), 男, 硕士研究生. E-mail: 946556446@qq.com

通讯作者：李 敏, 副教授. E-mail: limin9936@163.com; 杨 儒，教授.E-mail: ruyang@mail.buct.edu.cn 
化学性质, 而且还具有传统活性炭所不具有的天然 孔道结构与丰富的表面官能团 ${ }^{[3-4]}$ 。如今, 随着科研 工作者对生物质活性炭研究的日渐深入, 生物质活 性炭的应用范围已扩大至电池、超级电容器、电催 化、环境保护等各个领域 ${ }^{[5-6]}$ 。对生物质衍生的炭材 料进行了大量研究, 例如棉秆 ${ }^{[7]}$, 玉米秆 ${ }^{[8]}$ 和竹子 ${ }^{[9]}$ 等。Chen 等 ${ }^{[10]}$ 开创性地以天然木料为原料, 在保留 天然木料互联孔道结构的基础上制备出的全木活性 炭超级电容器电极材料性能优异, 远优于传统活性 炭。Yang 等 ${ }^{[11]}$ 以大麻茎为原料经原位炭化制备的 $\mathrm{C} / \mathrm{FeS} / \mathrm{Fe}$ 复合材料在工业除铬上表现优异。Hao 等 ${ }^{[2]}$ 以低成本的天然生物质棉花作为炭源制得氮掺杂多 孔炭材料, 为应用在超级电容器提供了可能。 $\mathrm{Yu}$ 等 ${ }^{[12]}$ 利用生物质 $\mathrm{D}$-葡萄糖和富氮三聚氰胺制备的 $\mathrm{N}$ 掺 杂多孔炭对 $\mathrm{H}_{2} \mathrm{~S}$ 的去除表现出优异的吸附催化性能, 在污染控制中有广阔的应用前景。

栓皮柇是取自于栓皮柇树根、茎部位的生物质 材料, 它由紧密排列的空腔细胞组成, 相邻的细胞 间被生物胶质紧密连接, 形成排列紧密的蜂窝状结 构 ${ }^{[13-15]}$ 。此外, 对栓皮栋的成分研究表明, 它由高炭 含量的木质素、纤维素、软木脂等构成, 是一种理 想的生物质活性炭原料 ${ }^{[16-17]}$ 。然而, 迄今为止, 栓皮 柇软木多数情况下只是被用于制作瓶塞, 其出色的 结构并未被充分利用, 甚至使用后还会被作为废料 焚烧，对环境造成污染。

本研究以充分利用栓皮柇软木为目的, 使用薄 壁组织组成的栓皮柇软木为前驱体, 在保留其原有 孔道结构的前提下，经清洗、破碎、预炭化、 $\mathrm{KOH}$ 高温活化等步骤, 控制实验条件, 制得了系列高比
表面积栓皮柇基活性炭, 并对其结构、形貌、表面 化学组成以及电化学性能进行了全面的研究。

\section{1 实验方法}

\section{1 试剂}

栓皮柇软木(取自于陕西，清洗后烘干备用); $\mathrm{KOH}$ (分析纯, 北京化工厂); $\mathrm{HCl}$ (分析纯, 北京化 工厂); $\mathrm{Na}_{2} \mathrm{SO}_{4}$ (分析纯, 北京通广精细化工)。

\section{2 栓皮栋软木基多孔活性炭片的制备}

软木经植物粉碎机粉碎, 取 $60 \sim 80 \mu \mathrm{m}$ 样品放 入管式炉, 升温速率为 $5{ }^{\circ} \mathrm{C} / \mathrm{min}$, 在 $\mathrm{N}_{2}$ 气氛下 $600{ }^{\circ} \mathrm{C}$ 炭化 $2 \mathrm{~h}$, 得栓皮柇软木炭; 以 $\mathrm{KOH} /$ 栓皮栎 软木炭质量比 $3.5,4.0,4.5,5.0$, 分别称量栓皮栋软 木炭 $1.5 \mathrm{~g}$, 对应 $\mathrm{KOH} 5.25,6.00,6.75,7.50 \mathrm{~g}$ 置于烧 杯中, 加入 $150 \mathrm{~mL}$ 去离子水, 加热摚拌至水蒸干, 放入干燥箱中干燥至恒重。将干燥产物置于管式炉 中, 升温速率为 $5{ }^{\circ} \mathrm{C} / \mathrm{min}$, 在 $\mathrm{N}_{2}$ 气氛下 $800{ }^{\circ} \mathrm{C}$ 活化 $3 \mathrm{~h}$ 。冷却后取出样品, 以盐酸中和剩余 $\mathrm{KOH}$, 随后 用去离子水洗涤、抽滤至中性, 将样品置于干燥箱 中干燥至恒重。干燥后的产物标记为 COAC- $n(n=$ $3.5,4.0,4.5,5.0), n$ 代表碱炭比。将未经 $\mathrm{KOH}$ 活化 的样品标记为 CC, 详细流程见图 1。

\section{3 样品表征}

热重分析(TG)采用德国耐驰公司的 TG 209 F3 型热重分析仪在氮气气氛下进行测试; 扫描电子显 微镜(SEM)测试采用日立 Hitachi S-4800 型扫描电 子显微镜; 高分辨透射电子显微镜(TEM)采用日本 JEOL JEM-3010 型透射电子显微镜; 采用美国
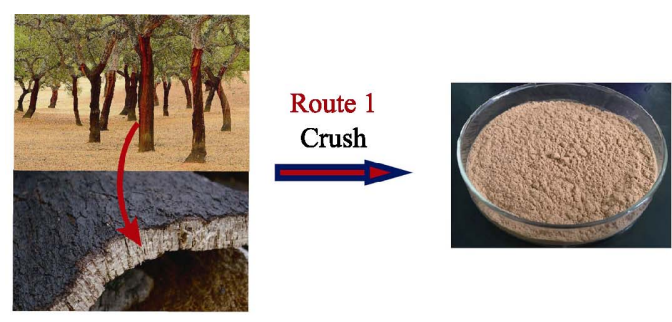

Carbonization
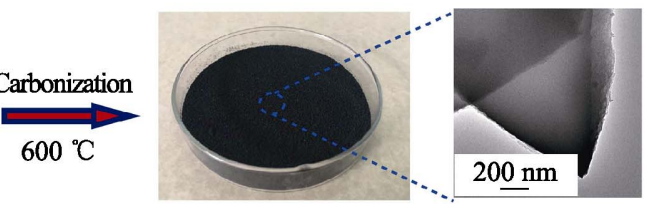

I

Cork-derived carbon
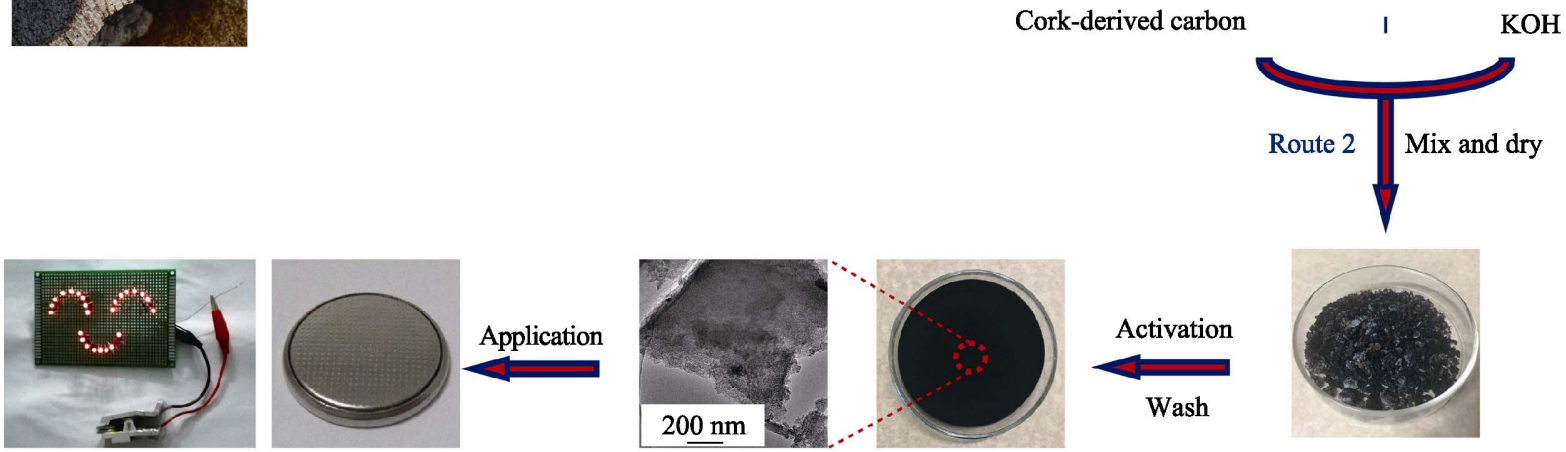

图 1 软木基多孔活性炭片制备流程及超级电容器应用

Fig. 1 Schematic illustration of the preparation process for cork-derived porous activated carbon sheets and application of supercapacitor 
Micromeritics ASAP2020 型表面积和孔隙率分析仪 对样品进行低温 $(77 \mathrm{~K}) \mathrm{N}_{2}$ 吸附脱附测试; 采用密度 泛函理论(Density Functional Theory, DFT)分析样品 孔径分布; 采用 BET 方法得到样品的比表面积; X 射线衍射 $(X R D)$ 测试采用浩元仪器 DX-2500 型 X 射 线衍射仪对样品进行分析, $\mathrm{CuK} \alpha(\lambda=0.1542 \mathrm{~nm})$ 作 为射线源; $X$ 射线光电子能谱(XPS) 采用 Thermo Scientific 公司 Escalab250 型 X 射线光电子能谱仪测 试, 采用单色 $\mathrm{MgK} \alpha(1253.6 \mathrm{eV})$ 作为射线源。

\section{4 电极制作及电容器组装}

循环伏安 $(\mathrm{CV})$ 、恒电流充放电与交流阻抗等电 化学性能测试在电化学工作站(上海辰华 CHI 760E) 与蓝电电池测试系统(LAND CT2001A)上进行。将 活性物质、乙炔黑与聚四氟乙烯(PTFE)按照 $8: 1: 1$ 的质量比充分混合, 辊压制成直径 $12 \mathrm{~mm}$ 的炭膜, $80{ }^{\circ} \mathrm{C}$ 干燥 $12 \mathrm{~h}$, 称重。用直径 $14 \mathrm{~mm}$ 的泡沫镍作为 集流器, 将炭膜在 $15 \mathrm{MPa}$ 压力下压在泡沫镍上形 成工作电极。然后, 以 $6 \mathrm{~mol} / \mathrm{L} \mathrm{KOH}$ 作为电解液, 录/ 氧化录电极作为参比电极, 铂片作为对电极, 与上 述制得的工作电极组装成三电极体系, 进行测试。 阻抗测试的频率范围为 $0.01 \mathrm{~Hz}$ 到 $100 \mathrm{kHz}$ 。质量比 电容通过以下公式计算:

$$
C_{s}=\frac{I \Delta t}{m \Delta V}
$$

其中, $I(\mathrm{~A})$ 为放电电流, $\Delta t(\mathrm{~s})$ 为放电时间, $m(\mathrm{~g}$ ) 为活 性物质质量, $\Delta V(\mathrm{~V})$ 为放电过程中除去电阻压降后 的电压变化。

为了进一步探究样品在实际应用中的价值, 组 装了两电极体系的对称型超级电容器。将上述制得 的质量相近的炭膜与经过超声处理的泡沫镍压在一 起(在 $15 \mathrm{MPa}$ 的压力下), 得到一组工作电极。把压 好的工作电极分别置于 $1 \mathrm{~mol} / \mathrm{L} \mathrm{Na}_{2} \mathrm{SO}_{4}$ 和 $6 \mathrm{~mol} / \mathrm{L}$ $\mathrm{KOH}$ 电解液中静置 $3 \mathrm{~h}$ 。分别以 $1 \mathrm{~mol} / \mathrm{L} \mathrm{Na}_{2} \mathrm{SO}_{4}$ 、 $6 \mathrm{~mol} / \mathrm{L} \mathrm{KOH}$ 为电解液, 聚丙烯膜为隔膜, 按照一 定顺序将其装入圆形不锈钢纽扣式电池壳(CR 2032 型) 中, 得到纽扣式超级电容器。单个电极的质量比 电容计算公式如下:

$$
C_{s}=\frac{2 I \Delta t}{m \Delta V}
$$

对称型超级电容器的能量密度 $E(\mathrm{Wh} / \mathrm{kg})$ 与功率密 度 $P(\mathrm{~W} / \mathrm{kg})$ 计算公式如下:

$$
\begin{gathered}
E=\frac{C_{S}(\Delta V)^{2}}{2 \times 4 \times 3.6} \\
P=\frac{E \times 3600}{\Delta t}
\end{gathered}
$$

\section{2 结果与讨论}

\section{1 栓皮栋软木原料的 TG/DTG 分析}

栓皮柇软木是由不同组分复合而成的天然聚合 物, 其主要化学组成为: 软木脂约 $40 \mathrm{wt} \%$, 木质素 约 $23 \mathrm{wt} \%$, 多糖约 $20 \mathrm{wt} \%$, 提取物约 $14 \mathrm{wt} \%$, 灰分 约 $1 \mathrm{wt} \%{ }^{[16]}$ 。图 2 是栓皮柇软木原料在 $\mathrm{N}_{2}$ 气氛下的 热解过程, 对于栓皮栋软木, 其热解主要发生在 200 500 ${ }^{\circ} \mathrm{C}$ 间, $200{ }^{\circ} \mathrm{C}$ 以下基本无质量损失, 表明栓 皮柇软木基本不含水分 ${ }^{[18]}$ 。栓皮柇软木在 200 $500{ }^{\circ} \mathrm{C}$ 间的热解主要涉及以下几个不同的热解反应: 在 200 330 ${ }^{\circ} \mathrm{C}$ 发生半纤维素的分解; 在 $330 \sim 400{ }^{\circ} \mathrm{C}$ 发生纤维素的分解 ${ }^{[19]}$; 在 $400 \sim 450{ }^{\circ} \mathrm{C}$ 与 330 500 ${ }^{\circ} \mathrm{C}$ 分别发生木质素与软木脂的分解, 该阶段软木脂分 解超过 $80 \%{ }^{[20-21]}$ 。以上总失重量共约占软木初始质 量的 76\%。根据 TG/ DTG 表征结果, 确定适宜的预 炭化温度为 $600{ }^{\circ} \mathrm{C}$ 。

\section{2 栓皮栋软木基多孔炭表面形貌分析}

图 3(a) (c) 是栓皮栋软木经 $600{ }^{\circ} \mathrm{C}$ 炭化后的样 品 CC 的 SEM 与 TEM照片, 由图 3(a)可以看出, 样 品 CC 为薄片状颗粒, 厚度 200 300 nm。图 3(b)是 其 TEM 照片, 从图中可以看出样品 CC 表面光滑、 密实。同时, 从样品 CC 的 TEM 照片中(图 3(c))可 以看到大量石墨片层的无序晶格条纹, 表明炭化后 的样品 CC 为密实的无定形炭材料。图 3(d) (f) 是样 品 $\mathrm{CC}$ 经 $800{ }^{\circ} \mathrm{C}$ 活化后制得的样品 COAC-4.5 的 $\mathrm{SEM}$ 与 $\mathrm{TEM}$ 照片, 从图 3(d)中可以看出活化后的 样品 COAC-4.5 保持了炭化样品 CC 原有的薄片状 形貌, 但对比样品 COAC-4.5(图 3(e)) 和样品 CC(图3(b)) 的 TEM 照片, 可以看出经 $\mathrm{KOH}$ 活化后, 样品 COAC-4.5 表面更为粗糙, 形成大量刻蚀孔，而未经 活化的样品 $\mathrm{CC}$ 表面十分光滑, 表明 $\mathrm{KOH}$ 对其起到 活化造孔作用, 这些孔结构可以为电荷的储存提供

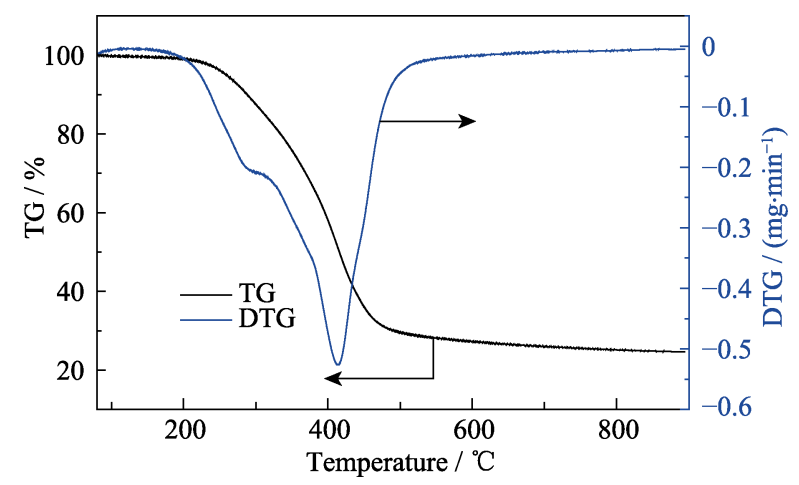

图 2 软木原料的 $\mathrm{TG} / \mathrm{DTG}$ 曲线

Fig. 2 TG/DTG curves of raw cork in a flow of nitrogen gas 
更多活性位点。由样品 COAC-4.5(图 3(f)) 和样品 $\mathrm{CC}$ (图 3(c)) 的 TEM 照片, 还可看出 $\mathrm{KOH}$ 的活化作 用使得样品 $\mathrm{CC}$ 的无定形晶体结构变得更加混乱, 这主要归因于 $\mathrm{KOH}$ 活化对其炭骨架的刻蚀作用, 形成了大量的孔结构。

\section{$2.3 \mathrm{~N}_{2}$ 吸附孔结构分析}

图 4(a) 是不同碱炭比下制得样品 COAC- $n$ 的 $\mathrm{N}_{2}$ 吸脱附等温线, 均为 I- 型。样品对氮气的吸附量在 相对压力 $\left(p / p_{0}\right)$ 较低时均呈现快速上升, 表明材料具 有丰富的微孔; 相对压力进一步升高时, 吸附量增 速明显减缓, 出现一个近似水平的平台, 表明材料 的孔结构以微孔为主。从图中曲线还可看出, 样品 在低相对压力时的吸附量随着碱炭比 $(\mathrm{KOH} / \mathrm{C})$ 的 升高而增大, 在碱炭比 $(\mathrm{KOH} / \mathrm{C})$ 为 4.5 时制得的样 品 COAC-4.5 具有最大吸附量, 表明增加 $\mathrm{KOH}$ 用量,
加强了活化作用, 使得孔结构进一步发育, 增加了 样品中的微孔。但是, 当 $\mathrm{KOH} / \mathrm{C}$ 比值升至 5.0 时, 样 品 COAC-5.0 在低相对压力处的吸附量略有下降, 表明 $\mathrm{KOH}$ 用量过多导致过度活化, 炭骨架坍塌, 孔 结构被破坏，微孔数量减少。

图 4(b) 是通过 DFT 法得到的孔径分布曲线, 结 合表 S1 中的孔结构参数可知, 碱炭比为 3.5 时, 样 品 COAC-3.5 的孔结构以 $0.5 \sim 1.0 \mathrm{~nm}$ 的超微孔为主, 含有少量 1 2 $\mathrm{nm}$ 的微孔, 基本不含介孔。随碱炭比 的升高, 可以明显看到样品孔径分布快速拓宽, 其 中 1 2 $\mathrm{nm}$ 的微孔快速增多, 且出现大量 2 4 $\mathrm{nm}$ 的 介孔。1 2 $\mathrm{nm}$ 的微孔及 $2 \sim 4 \mathrm{~nm}$ 的介孔的引入有利 于离子在炭材料内部的扩散, 为离子提供更短的快 速扩散通道, 从而有利于样品倍率性能的提高, 而 $0.5 \sim 1.0 \mathrm{~nm}$ 的超微孔则为电荷的堆积提供更多的活
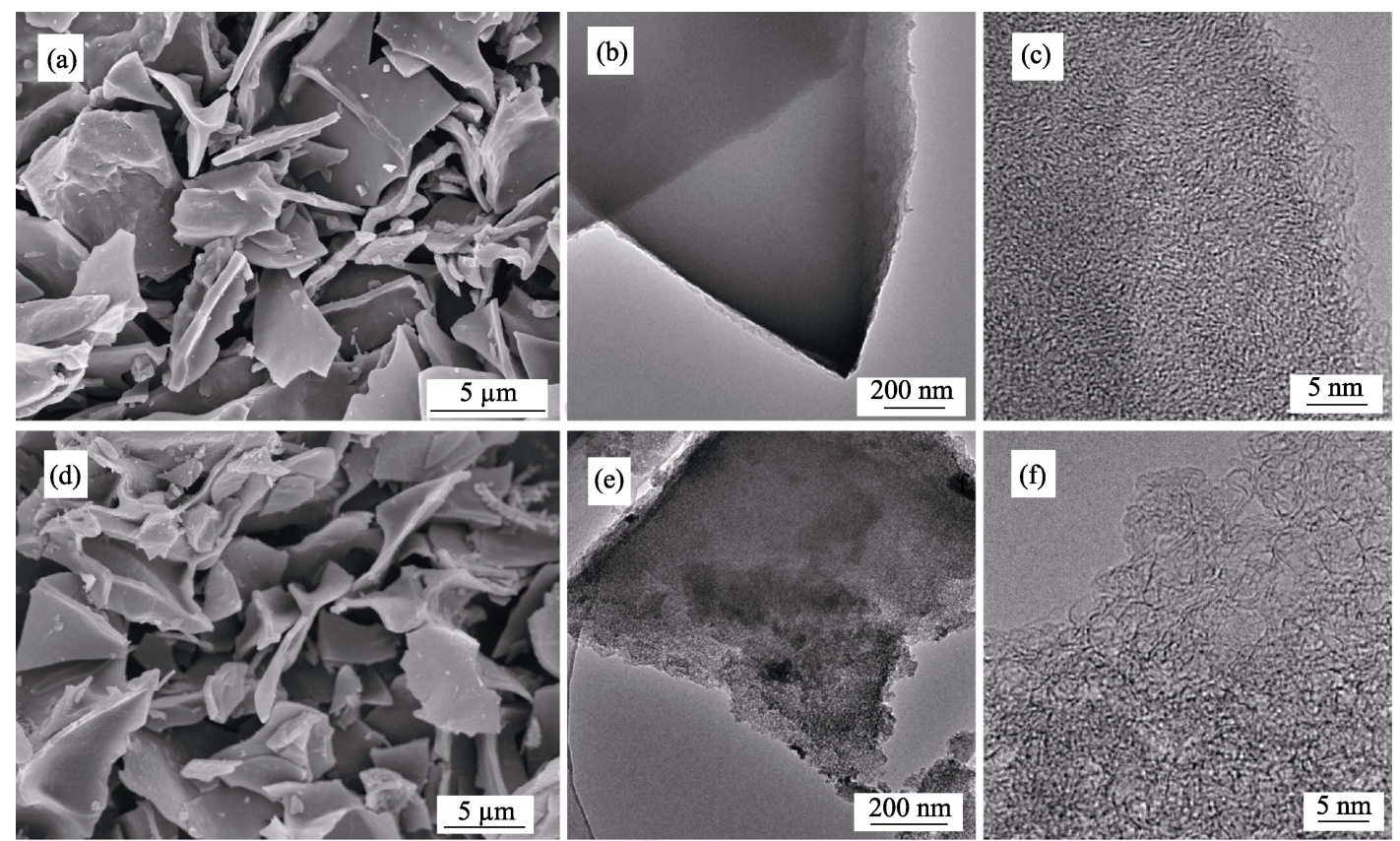

图 3 (a) (c)样品 CC 和(d) (f)样品 COAC-4.5 的(a, d) SEM 和(b, c, e, f)TEM 照片

Fig. 3 (a, d) SEM and (b, c, e, f) TEM images of (a-c) CC and (d-f) COAC-4.5
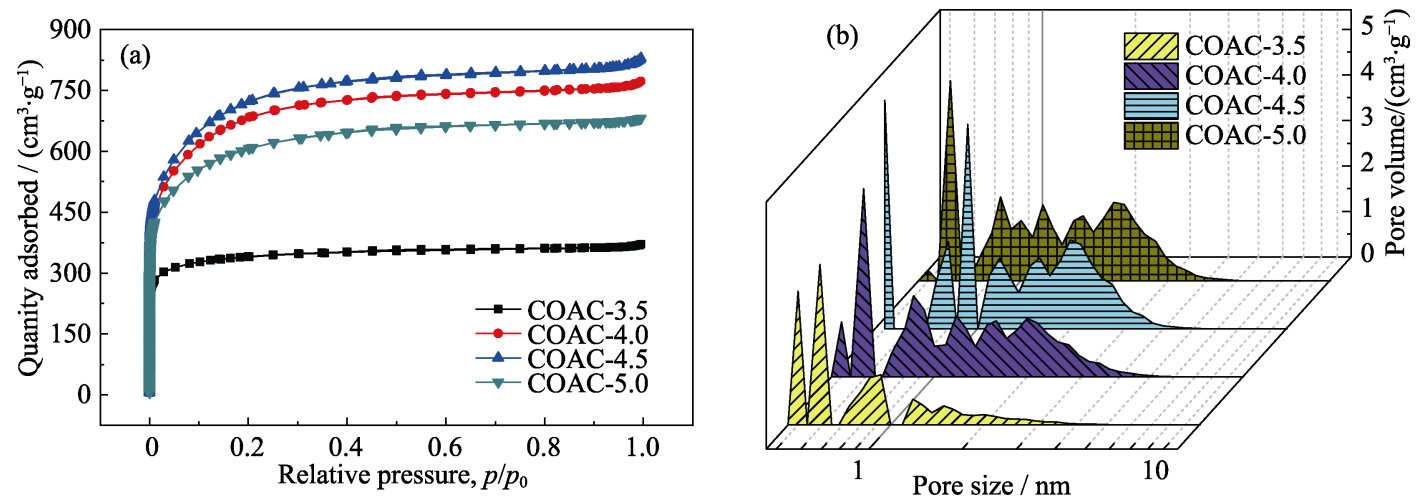

图 4 样品 COAC- $n$ 的(a) $\mathrm{N}_{2}(77 \mathrm{~K})$ 吸脱附等温线和(b)孔径分布曲线

Fig. 4 (a) $\mathrm{N}_{2}(77 \mathrm{~K}$ ) adsorption/desorption isotherms and (b) pore size distribution curves of COAC- $n$ samples 
性位点。由表 S1 中的样品 COAC- $n$ 的孔结构参数 可知, 在碱炭比为 4.5 时制得的样品 COAC- 4.5 具有 最大的比表面积和总孔容, 分别为 $2312 \mathrm{~m}^{2} / \mathrm{g}$ 和 $1.28 \mathrm{~cm}^{3} / \mathrm{g}$, 其超高的比表面积有利于堆积更多的 电解液离子，从而提高栓皮栋软木基多孔活性炭片 的电容量。

\section{4 栓皮栋软木基多孔炭 XRD 分析}

图 5 是不同碱炭比下制得样品的 XRD 图谱。 从图中可以看出, 四个样品的衍射曲线在约 $2 \theta=$ $23^{\circ}$ 和 $43.8^{\circ}$ 处均有两个宽衍射峰, 分别对应于石墨 炭的(002)晶面和(101)晶面 ${ }^{[22]}$ 。相比样品 COAC-4.0、 COAC-4.5 和 COAC-5.0, 样品 COAC-3.5 的这两个特 征峰更加尖锐, 表明具有相对较高的石墨化程度。

随着碱炭比增至 4.0、4.5 和 5.0 时, 活化后样品 的衍射峰明显减弱, 表明由于 $\mathrm{KOH}$ 用量的升高, 活 化刻蚀强度增强，整齐的石墨层随碱炭比升高而遭 到破坏, 炭结构的有序度降低, 缺陷增多, 石墨化 程度减弱。

\section{5 栓皮栋软木基多孔炭的 XPS 分析}

图 6(a)是不同碱炭比制得样品的 XPS 图谱, 在 284.6 与 $533 \mathrm{eV}$ 处出现两个明显的峰, 分别对应 $\mathrm{C}$ $1 \mathrm{~s}$ 与 $\mathrm{O} 1 \mathrm{~s}$ 峰, 在 $401 \mathrm{eV}$ 处较弱的峰对应 $\mathrm{N} 1 \mathrm{~s}$ 峰。 样品的 C、N、O 元素的具体含量见表 $\mathrm{S} 2$, 样品 COAC-4.5 的 $\mathrm{N} 、 \mathrm{O}$ 元素含量分别为 $1.34 \mathrm{at} \%$ 、 $12.54 \mathrm{at} \%$ 。图 6(b)为样品 COAC-4.5 的 C 1s 分峰图 谱, 284.6、285.6、286.7 和 $289.2 \mathrm{eV}$ 处的峰对应 $\mathrm{C}$ 元素不同的化学态 $\mathrm{sp}^{2}-\mathrm{C} 、 \mathrm{sp}^{3}-\mathrm{C} 、 \mathrm{C}-\mathrm{O} 、 \mathrm{C}=\mathrm{O}^{[23-24]}$ 。 图 6(c)是样品 COAC-4.5 的 O 1s 分峰图谱, $\mathrm{O}$ 原子 的化学状态可以分为三类, $\mathrm{O}-\mathrm{I}: 531.7 \mathrm{eV}$ 处 $-\mathrm{C}=\mathrm{O}$ 基团; O-II: $533 \mathrm{eV}$ 处-C-OH/C-O-C 基团; O-III: $534.5 \mathrm{eV}$ 处化学吸附水或氧 ${ }^{[25]}$ 。这些丰富的含氧基 团可以增强样品的亲水性, 从而扩大活性表面面积, 并能产生额外的噟电容, 提高材料的整体电容量 ${ }^{[26-27]}$ 。

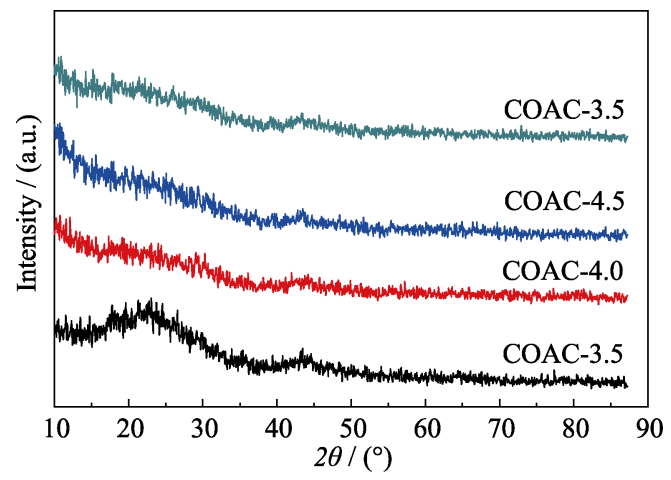

图 5 样品 $\mathrm{COAC}-n$ 的 XRD 图谱

Fig. 5 XRD patterns of the COAC- $n$ samples
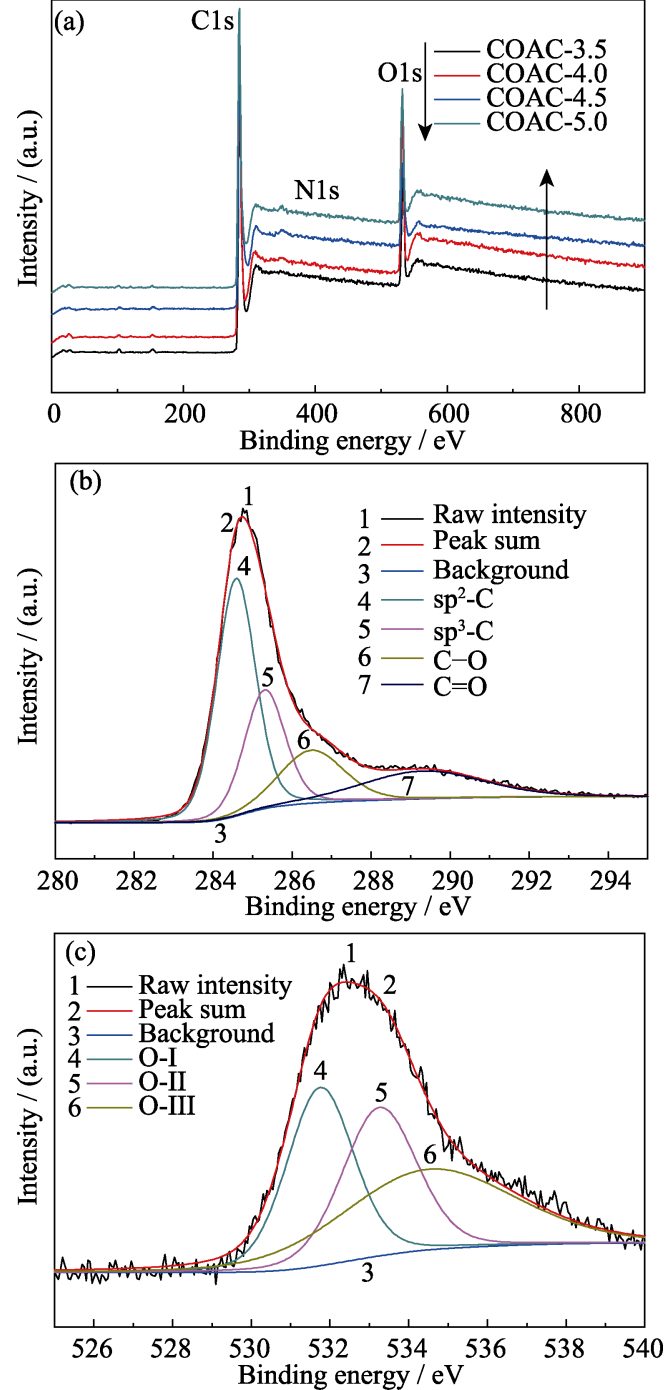

图 6 (a)样品 COAC- $n$ 的 XPS 图谱, 样品 COAC-4.5 的 (b)C1s 图谱和(c)O1s 图谱

Fig. 6 (a) XPS spectra of COAC- $n$, (b) C1s and (c) O1s XPS spectra of COAC-4.5

\section{6 三电极电化学性能分析}

图 7 是样品 $\mathrm{COAC}-n$ 在 $\mathrm{KOH}$ 三电极体系中的 电化学性能。图 7(a)和(b) 是扫描速率为 1 和 $200 \mathrm{mV} / \mathrm{s}$ 时样品的 $\mathrm{CV}$ 曲线, 电势窗口为 $-1.0 \mathrm{~V} \sim 0$ 。从图中可 以看出, $\mathrm{CV}$ 曲线为典型的类矩形曲线, 表明样品的 电容性能为典型的双电层电容; 在 $-1 \sim-0.5 \mathrm{~V}$ 范围 内存在较宽的氧化还原峰, 表明样品还含有赝电容, 这归因于样品表面丰富的杂原子可以进行快速氧化 还原反应 ${ }^{[28-29]}$, 反应机理如式(5) (9)所示：

$$
\begin{gathered}
>\mathrm{C}-\mathrm{OH}+\mathrm{OH}^{-} \Leftrightarrow>\mathrm{CO}^{-}+\mathrm{H}_{2} \mathrm{O} \\
>\mathrm{C}=\mathrm{O}+\mathrm{OH}^{-} \Leftrightarrow-\mathrm{COOH}+\mathrm{e}^{-} \\
-\mathrm{COOH}+\mathrm{OH}^{-} \Leftrightarrow-\mathrm{COO}^{-}+\mathrm{H}_{2} \mathrm{O}
\end{gathered}
$$

$>\mathrm{CH}-\mathrm{NH}_{2}+2 \mathrm{OH}^{-} \Leftrightarrow \mathrm{C}=\mathrm{NH}+2 \mathrm{H}_{2} \mathrm{O}+2 \mathrm{e}^{-}$

$>\mathrm{CH}-\mathrm{NH}_{2}+2 \mathrm{OH}^{-} \Leftrightarrow>\mathrm{C}-\mathrm{NHOH}+\mathrm{H}_{2} \mathrm{O}+2 \mathrm{e}^{-}$ 

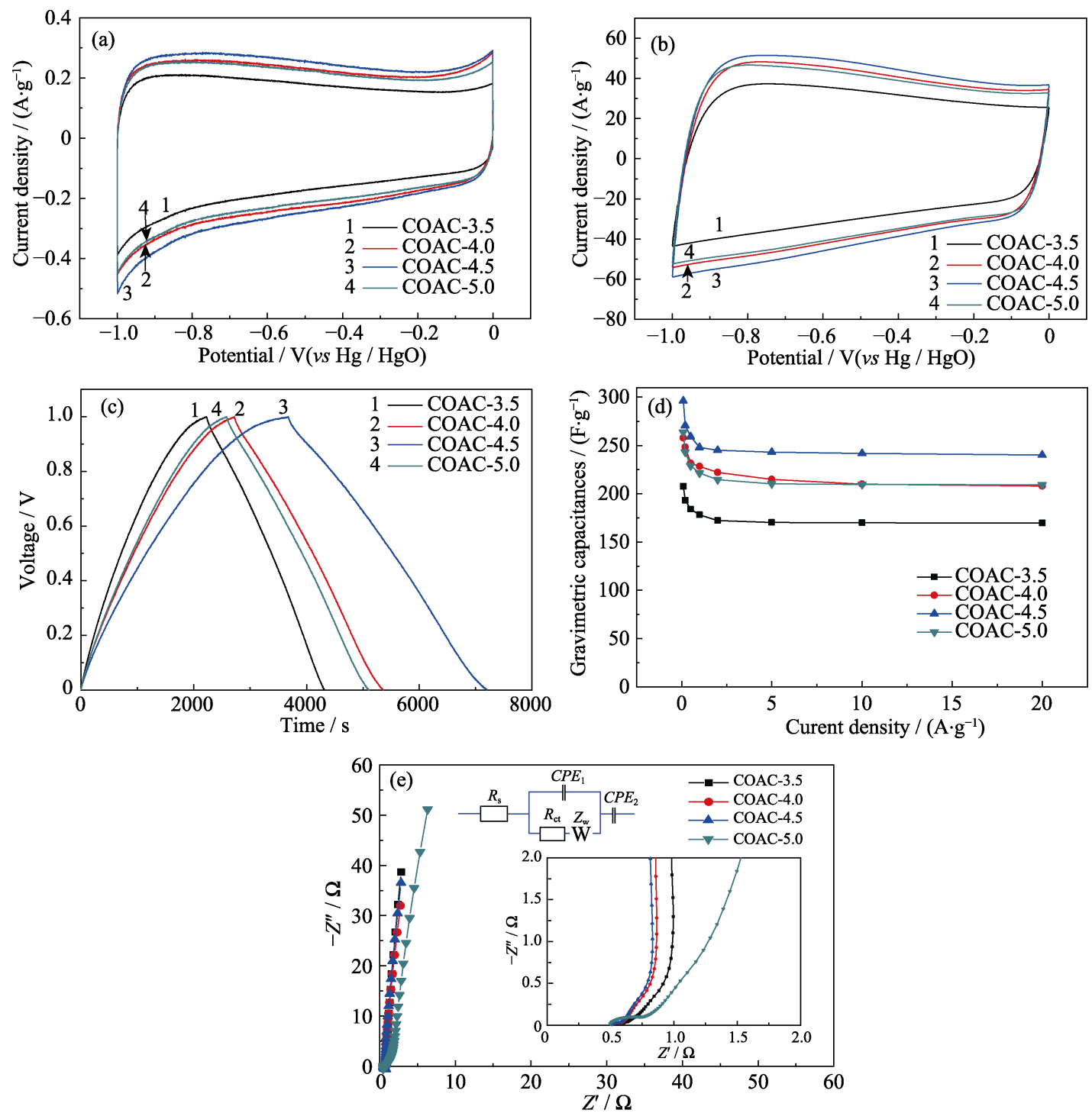

图 7 样品 COAC- $n$ 在 $6 \mathrm{~mol} / \mathrm{L} \mathrm{KOH}$ 三电极体系的电化学性能: 在扫描速率(a) 1 和 $200 \mathrm{mV} / \mathrm{s}(\mathrm{b})$ 下的循环伏安曲线;

(c)在电流密度 $0.1 \mathrm{~A} / \mathrm{g}$ 下的恒电流充放电曲线; (d) 比电容与电流密度函数关系曲线; (e) Nyquist 图谱

Fig. 7 Electrochemical performance characteristics of COAC- $n$ measured in a three-electrode system in the $6 \mathrm{~mol} / \mathrm{L} \mathrm{KOH}$ electrolyte: CV curves at (a) $1 \mathrm{mV} / \mathrm{s}$ and (b) $200 \mathrm{mV} / \mathrm{s}$; (c) Galvanostatic charge/discharge curves at a current density of $0.1 \mathrm{~A} / \mathrm{g}$;

(d) Specific capacitances at different current densities; (e) Nyquist plots in the frequency range from $10 \mathrm{kHz}$ to $10 \mathrm{mHz}$ with inset showing magnified figure of arc part

在扫描速率为 $200 \mathrm{mV} / \mathrm{s}$ 时, 样品仍保持非常好 的近矩形形状, 表明样品具有良好的倍率特性。样 品 COAC-4.5 的 CV 曲线的环绕面积最大, 表明其具 有最高的电容量, 主要归因于其最高的比表面积 $\left(2312 \mathrm{~m}^{2} / \mathrm{g}\right)$, 为电解液离子的堆积提供了更多的活 性位点。图 7(c) 是所有样品在 $0.1 \mathrm{~A} / \mathrm{g}$ 电流密度下的 $\mathrm{GCD}$ 曲线, 曲线为有轻微扭曲的对称三角形, 表明 电容以双电层电容为主。但其放电曲线稍有扭曲, 表明还存在噟电容, 这与 $\mathrm{CV}$ 曲线结果一致。图 7(d) 是各样品在 $0.1 \sim 20 \mathrm{~A} / \mathrm{g}$ 电流密度下的比电容曲线, 从图中可以看出, 在不同电流密度下样品 $\mathrm{COAC}$ 4.5 的比电容均大于其他样品, 在 $0.1 \mathrm{~A} / \mathrm{g}$ 时比电容 高达 $296 \mathrm{~F} / \mathrm{g}$, 且在电流密度增至 $20 \mathrm{~A} / \mathrm{g}$ 时, 仍保持
$240 \mathrm{~F} / \mathrm{g}$, 电容保持率达到 $81.1 \%$, 表现出极佳的倍 率性能, 这主要归因于其炭颗粒薄片状结构(厚度 200 300 nm) 以及引入大量微孔、介孔(1 4 nm) 为离 子扩散提供了快速通道。

图 7(e) 是各样品在 $0.01 \mathrm{~Hz}$ 到 $10 \mathrm{kHz}$ 频率范围 内的 Nyquist 曲线。如图 7(e)所示, 低频部分接近垂 直的曲线代表电极材料内较小的电解液离子扩散电 阻和极好的电容性能。样品 COAC-3.5、COAC-4.0、 COAC-4.5 和 COAC-5.0 的 Nyquist 曲线在低频区与 实轴近乎垂直, 均具有极好的电容性能, 内部孔道 结构疏通。高频区 $Z^{\prime}$ 轴的截距代表等效串联电阻 $\left(R_{\mathrm{s}}\right)$, 包括电解液电阻、活性物质固有电阻以及集流器与 活性物质之间连接电阻的总和。从图 7(e)中可见样 
品 COAC-3.5、COAC-4.0、COAC-4.5 和 COAC-5.0 的 $R_{\mathrm{s}}$ 值均较小, 约为 $0.5 \Omega$, 表明各样品均具有较好 的导电性。半圆区代表了电解液与活性物质连接界 面的电荷转移过程, 较小的半圆表明电荷转移阻抗 较小; 中频区域的 Warburg-型曲线(斜率 $45^{\circ}$ )代表离 子由电解液扩散至电极材料表面的阻抗; 从图中可 以看出样品 COAC-4.5 具有最小的电荷转移阻抗和 最快的离子扩散速率, 该结果与上述测试结果一致。

\section{7 两电极电化学性能分析}

以 $6 \mathrm{~mol} / \mathrm{L} \mathrm{KOH}$ 为电解液, 组装两电极超级电 容器, 评价样品 COAC-4.5 作为超级电容器电极材 料的实际应用价值。图 S1(a)和(b)非常近似矩形的 循环伏安曲线表明基于样品 COAC-4.5 的超级电容 器具有极好的双电层电容特性。图 S1(c)为恒电流充 放电曲线, $\mathrm{COAC}-4.5 / / \mathrm{COAC}-4.5$ 超级电容器在电流 密度大于 $10 \mathrm{~A} / \mathrm{g}$ 时仍保持非常好的对称三角形形状, 表明了其极佳的倍率性能; 在电流密度为 $20 \mathrm{~A} / \mathrm{g}$ 时 欧姆降仅为 $53 \mathrm{mV}$, 表明组装的超级电容器整体内 阻较小。图 S1(d)是不同电流密度下的 COAC-4.5// COAC-4.5 超级电容器的单电极比电容曲线, 当电 流密度由 $1 \mathrm{~A} / \mathrm{g}(212 \mathrm{~F} / \mathrm{g})$ 增至 $50 \mathrm{~A} / \mathrm{g}(160 \mathrm{~F} / \mathrm{g})$ 时, 得 到的电容保持率达到 $75.5 \%$, 表明电解质离子可以 在高电流密度下高效、快速地到达样品 COAC-4.5 的电化学活性界面。图 S1(e)是 $6 \mathrm{~mol} / \mathrm{L} \mathrm{KOH}$ 电解液 两电极体系下的能量比较图, 从图中可以看出能量 密度随功率密度增加而降低。在功率密度为 $25 \mathrm{~W} / \mathrm{kg}$ 时能量密度达到最大, $8.57 \mathrm{Wh} / \mathrm{kg}$, 且在功率密度为 $4748 \mathrm{~W} / \mathrm{kg}$ 时能量密度仍能保持 $5.42 \mathrm{Wh} / \mathrm{kg}$, 表明 随着功率密度的增加能量损失较小。图 S1(f) 是 COAC-4.5//COAC- 4.5 超级电容器在电流密度 $5 \mathrm{~A} / \mathrm{g}$ 下循环 5000 次的比电容变化, 在循环 5000 次后电 容保持率高达 $99.5 \%$, 表现出极好的循环稳定性。 根据能量公式 $E=1 / 2 C V^{2}$ 可知, 所组装的对称型超级 电容器的能量容量与其工作电压窗口成正比, 即工 作电压窗口越大其能够储存的能量越多。已知 $\mathrm{Na}_{2} \mathrm{SO}_{4}$ 电解液具有比 $\mathrm{KOH}$ 电解液更高的工作电压 窗口 ${ }^{[30]}$, 可以进一步提高基于样品 COAC-4.5 超级 电容器的能量密度。以 $1 \mathrm{~mol} / \mathrm{L} \mathrm{Na}_{2} \mathrm{SO}_{4}$ 为电解液, 组 装对称型超级电容器, 进一步评价 COAC-4.5 电极 的超级电容性能。图 S2(a) 是在扫描速率 $50 \mathrm{mV} / \mathrm{s}$ 下、 不同电势窗口的循环伏安曲线, 从图中可以看出, 当工作电压窗口增至 $1.8 \mathrm{~V}$ 时所得循环伏安曲线仍 保持矩形, 在阳极电流下无明显变形, 这表明电解 液没有分解, 主要是由于 $\mathrm{Na}_{2} \mathrm{SO}_{4}$ 电解液中 $\mathrm{H}^{+}$或 $\mathrm{OH}^{-}$较少, 使样品 COAC-4.5 表面氢离子的堆积低
于水分解的热力学电势 ${ }^{[31]}$ 。图 S2(b) (d) 展现出样品 具有以下三点特性：(1)理想的电容性，电解液离子 可以快速扩散至电极材料表面，且欧姆降较小表明 其具有较小的整体内阻; (2)极佳的倍率性能; (3)较 高的能量密度, 可以同时点亮 25 个 $3 \mathrm{~V}$ 红色 LED 灯(图 1 所示)。这一结果优于最近报道的 $\mathrm{Na}_{2} \mathrm{SO}_{4}$ 电 解液体系下的炭基对称型超级电容器 ${ }^{[32-34]}$ 。综上所 述, 以 $1 \mathrm{~mol} / \mathrm{L} \mathrm{Na}_{2} \mathrm{SO}_{4}$ 为电解液组装的 COAC-4.5// COAC-4.5 超级电容器具有极佳的电化学性能, 这 主要归因于以下三方面因素: 首先, 样品 COAC-4.5 具有最大的比表面积, 为电荷的储存提供了大量活 性位点; 其次, 样品 COAC-4.5 的孔尺寸大于电解 液离子尺寸 $\left(\mathrm{Na}^{+} \text {约 } 0.095 \mathrm{~nm}, \mathrm{SO}_{4}{ }^{2-} \text { 约 } 0.29 \mathrm{~nm}\right)^{[35]}$, 避免了离子堵塞现象, 有利于离子在材料内部的扩 散; 最后, 样品 COAC-4.5 薄片状的结构 (厚度 200 300 nm)、大量微孔及介孔 $(1 \sim 4 \mathrm{~nm})$ 的引入为离 子的扩散提供了更短的快速通道。因此, 在碱炭比 为 4.5 时所制备的样品 COAC-4.5 是一种良好的电 极材料, 适用于制作高能量、高功率、低成本的超 级电容器。

\section{3 结论}

以栓皮栋软木为原料, 经 $600{ }^{\circ} \mathrm{C}$ 预炭化后，以 $\mathrm{KOH}$ 为活化剂在 $800{ }^{\circ} \mathrm{C}$ 活化制得栓皮柇软木基多 孔活性炭。样品呈薄片状(厚度 200 300 nm), 丰富 的超微孔以及大量的微孔及介孔(1 4 nm), 其 BET 比表面积最大可达 $2312 \mathrm{~m}^{2} / \mathrm{g}$ 。在 $\mathrm{KOH}$ 三电极体系 中, 样品在 $0.1 \mathrm{~A} / \mathrm{g}$ 电流密度下比电容可达 $296 \mathrm{~F} / \mathrm{g}$; 在 $20 \mathrm{~A} / \mathrm{g}$ 电流密度下依然保持 $240 \mathrm{~F} / \mathrm{g}$ 的比电容, 倍 率性能良好。在 $\mathrm{KOH}$ 两电极体系中, 样品的单电极 比电容达 $247 \mathrm{~F} / \mathrm{g}$, 循环 5000 次, 电容保持率达 $99.5 \%$ ，表现出极好的循环稳定性。以 $1 \mathrm{~mol} / \mathrm{L}$ $\mathrm{Na}_{2} \mathrm{SO}_{4}$ 为电解液, 组装的对称型超级电容器能量密 度达 $19.62 \mathrm{Wh} / \mathrm{kg}$; 在 $50 \mathrm{~A} / \mathrm{g}$ 时, 电容保持率可达 $80.5 \%$ ，表现出优异的倍率性能。所制得的软木基多 孔活性炭片有望用于制备高能量、高功率的超级电 容器，在超级电容器领域具有良好的应用前景。

\section{参考文献:}

[1] DE WIT M, FAAIJ A. European biomass resource potential and costs. Biomass and Bioenergy, 2010, 34(2): 188-202.

[2] HAO Y X, QIAN M, XU J J, et al. Synthesis, microstructure and superconductivity of cotton-based porous carbon materials. Journal of Inorganic Materials, 2018, 33(1): 93-99.

[3] SEVILLA M, MOKAYA R. Energy storage applications of activated carbons: supercapacitors and hydrogen storage. Energy \& 
Environmental Science, 2014, 7(4): 1250-1280.

[4] WICKRAMARATNE N P, XU J, WANG M, et al. Nitrogen enriched porous carbon spheres: attractive materials for supercapacitor electrodes and $\mathrm{CO}_{2}$ adsorption. Chemistry of Materials, 2014, 26(9): 2820-2828.

[5] ZHAI Y, DOU Y, ZHAO D, et al. Carbon materials for chemical capacitive energy storage. Advanced Materials, 2011, 23(42): 4828-4850.

[6] ZHANG L L, ZHAO X S. Carbon-based materials as supercapacitor electrodes. Chemical Society Reviews, 2009, 38(9): 2520-2531.

[7] TIAN X, MA H, LI Z, et al. Flute type micropores activated carbon from cotton stalk for high performance supercapacitors. Journal of Power Sources, 2017, 359: 88-96.

[8] YU K, ZHU H, QI H, et al. High surface area carbon materials derived from corn stalk core as electrode for supercapacitor. Diamond and Related Materials, 2018, 88: 18-22.

[9] YANG C S, JANG Y S, JEONG H K. Bamboo-based activated carbon for supercapacitor applications. Curr. Appl. Phys., 2014, 14(12): 1616-1620.

[10] CHEN C, ZHANG Y, LI Y, et al. All-wood, low tortuosity, aqueous, biodegradable supercapacitors with ultra-high capacitance. Energy \& Environmental Science, 2017, 10(2): 538-545.

[11] YANG R, WANG Y, LI M, et al. A new carbon/ferrous sulfide/ iron composite prepared by an in situ carbonization reduction method from hemp (Cannabis sativa L.) stems and its $\mathrm{Cr}$ (VI) removal ability. ACS Sustainable Chemistry \& Engineering, 2014, 2(5): 1270-1279.

[12] YU Z F, WANG X Z, HOU Y N, et al. Preparation of nitrogendoped porous carbon by molten salt method and its catalytic desulfurization performance. Journal of Inorganic Materials, 2017, 32(7): 770-776.

[13] ATANES E, NIETO-MÁRQUEZ A, CAMBRA A, et al. Adsorption of $\mathrm{SO}_{2}$ onto waste cork powder-derived activated carbons. Chemical Engineering Journal, 2012, 211(22): 60-67.

[14] JAIN A, BALASUBRAMANIAN R, SRINIVASAN M P. Production of high surface area mesoporous activated carbons from waste biomass using hydrogen peroxide-mediated hydrothermal treatment for adsorption applications. Chemical Engineering Journal, 2015, 273: 622-629.

[15] KIM M J, JUNG M J, KIM M I, et al. Adsorption characteristics of toluene gas using fluorinated phenol-based activated carbons. Applied Chemistry for Engineering, 2015, 26(5): 587-592.

[16] ROSAS J M, RUIZ-ROSAS R, RODRÍGUEZ-MIRASOL J, et al. Kinetic study of $\mathrm{SO}_{2}$, removal over lignin-based activated carbon. Chemical Engineering Journal, 2017, 307: 707-721.

[17] LULAI E C, CORSINI D L. Differential deposition of suberin phenolic and aliphatic domains and their roles in resistance to infection during potato tuber (Solanum tuberosum L.) wound-healing. Physiological and Molecular Plant Pathology, 1998, 53(4): 209-222.

[18] PILAO R, RAMALHO E, PINHO C. Overall characterization of cork dust explosion. Journal of Hazardous Materials, 2006, 133(1): 183-195.

[19] YANG H P, YAN R, CHEN H P, et al. Characteristics of hemicellulose, cellulose and lignin pyrolysis. Fuel, 2007, 86(12):
1781-1788.

[20] CORDEIRO N, BELGACEM N M, GANDINI A, et al. Cork suberin as a new source of chemicals: 2. Crystallinity, thermal and rheological properties. Bioresource Technology, 1998, 63(2): 153158.

[21] JIANG G, NOWAKOWSKI D J, BRIDGWATER A V. A systematic study of the kinetics of lignin pyrolysis. Thermochimica Acta, 2010, 498(1): 61-66.

[22] JOHN M J, THOMAS S. Biofibres and biocomposites. Carbohydrate Polymers, 2008, 71(3): 343-364.

[23] RAYMUNDO-PIÑERO E, LEROUX F, BÉGUIN F. A highperformance carbon for supercapacitors obtained by carbonization of a seaweed biopolymer. Advanced Materials, 2006, 18(14): 1877-1882.

[24] LI Y, ZHAO Y, CHENG H, et al. Nitrogen-doped graphene quantum dots with oxygen-rich functional groups. Journal of the American Chemical Society, 2011, 134(1): 15-18.

[25] BINIAK S, SZYMAŃSKI G, SIEDLEWSKI J, et al. The characterization of activated carbons with oxygen and nitrogen surface groups. Carbon, 1997, 35(12): 1799-1810.

[26] ZHAO Y F, RAN W, HE J, et al. Oxygen-rich hierarchical porous carbon derived from artemia cyst shells with superior electrochemical performance. ACS Applied Materials \& Interfaces, 2015, 7(2): 1132-1139.

[27] ZHU H, WANG X L, YANG F, et al. Promising carbons for supercapacitors derived from fungi. Advanced Materials, 2011, 23(24): 2745-2748.

[28] JIANG L, SHENG L, LONG C, et al. Functional pillared graphene frameworks for ultrahigh volumetric performance supercapacitors. Advanced Energy Materials, 2015, 5(15): 1500771-1-9.

[29] XU B, HOU S, CAO G, et al. Sustainable nitrogen-doped porous carbon with high surface areas prepared from gelatin for supercapacitors. Journal of Materials Chemistry, 2012, 22(36): 1908819093.

[30] ZHONG C, DENG Y D, HU W B, et al. A review of electrolyte materials and compositions for electrochemical supercapacitors. Chemical Society Reviews, 2015, 44(21): 7484-7539.

[31] YU A, CHABOT V, ZHANG J. Electrochemical supercapacitors for energy storage and delivery: fundamentals and applications. CRC Press, 2013.

[32] WANG Q, YAN J, WANG Y B, et al. Three-dimensional flower-like and hierarchical porous carbon materials as high-rate performance electrodes for supercapacitors. Carbon, 2014, 67(2): $119-127$.

[33] SUN G L, LI B, RAN J B, et al. Three-dimensional hierarchical porous carbon/graphene composites derived from graphene oxidechitosan hydrogels for high performance supercapacitors. Electrochimica Acta, 2015, 171: 13-22.

[34] LONG C L, CHEN X, JIANG L L, et al. Porous layer-stacking carbon derived from in-built template in biomass for high volumetric performance supercapacitors. Nano Energy, 2015, 12: 141151.

[35] YAN, J, WANG, Q, WEI T, FAN Z J. Recent advances in design and fabrication of electrochemical supercapacitors with high energy densities. Adv. Energy Mater., 2014, 4(4): 1300816-1-43. 


\title{
补充材料:
}

\section{用于高性能超级电容器电极的栓皮栋基多孔活性炭的制备}

\author{
许伟佳，邱大平，刘诗强，李 敏，杨 儒
}

(北京化工大学 材料电化学过程与技术北京市重点实验室, 化工资源有效利用国家重点实验室, 北京 100029)

表 S1 样品 COAC-n 的孔结构参数

Table S1 Porosity parameters of the COAC-n samples

\begin{tabular}{|c|c|c|c|c|c|c|c|c|c|}
\hline \multirow[b]{2}{*}{ Samples } & \multirow{2}{*}{$\begin{array}{c}S_{\mathrm{BET}} / \\
\left(\mathrm{m}^{2} \cdot \mathrm{g}^{-1}\right)\end{array}$} & \multirow{2}{*}{$\begin{array}{c}{ }^{\mathrm{a}} D_{\text {ave }} / \\
\mathrm{nm}\end{array}$} & \multirow{2}{*}{$\begin{array}{c}V_{\mathrm{t}} / \\
\left(\mathrm{cm}^{3} \cdot \mathrm{g}^{-1}\right)\end{array}$} & \multicolumn{6}{|c|}{ DFT Method } \\
\hline & & & & $\begin{array}{c}S_{<1 \mathrm{~nm}} / \\
\left(\mathrm{m}^{2} \cdot \mathrm{g}^{-1}\right)\end{array}$ & $\begin{array}{l}S_{1-2 \mathrm{~nm}} / \\
\left(\mathrm{m}^{2} \cdot \mathrm{g}^{-1}\right)\end{array}$ & $\begin{array}{l}S_{2-4 \mathrm{~nm}} / \\
\left(\mathrm{m}^{2} \cdot \mathrm{g}^{-1}\right)\end{array}$ & $\begin{array}{c}V_{<1 \mathrm{~nm}} / \\
\left(\mathrm{cm}^{3} \cdot \mathrm{g}^{-1}\right)\end{array}$ & $\begin{array}{c}V_{1-2 \mathrm{~nm}} / \\
\left(\mathrm{cm}^{3} \cdot \mathrm{g}^{-1}\right)\end{array}$ & $\begin{array}{c}V_{2-4 \mathrm{~nm}} / \\
\left(\mathrm{cm}^{3} \cdot \mathrm{g}^{-1}\right)\end{array}$ \\
\hline COAC-3.5 & 1044 & 2.19 & 0.57 & 1097 & 115 & 29 & 0.34 & 0.08 & 0.04 \\
\hline COAC-4.0 & 2169 & 2.20 & 1.19 & 975 & 554 & 221 & 0.33 & 0.37 & 0.26 \\
\hline COAC-4.5 & 2312 & 2.22 & 1.28 & 1191 & 485 & 247 & 0.40 & 0.35 & 0.29 \\
\hline COAC-5.0 & 1929 & 2.18 & 1.05 & 1087 & 423 & 172 & 0.36 & 0.29 & 0.21 \\
\hline
\end{tabular}

Note: Average pore size $D_{\text {ave }}$ is calculated by BET method. $D_{\text {ave }}=4 V / A . V$ represents total pore volume $V_{\mathrm{t}}$ of single point adsorption. $A$ represents specific surface area of BET.
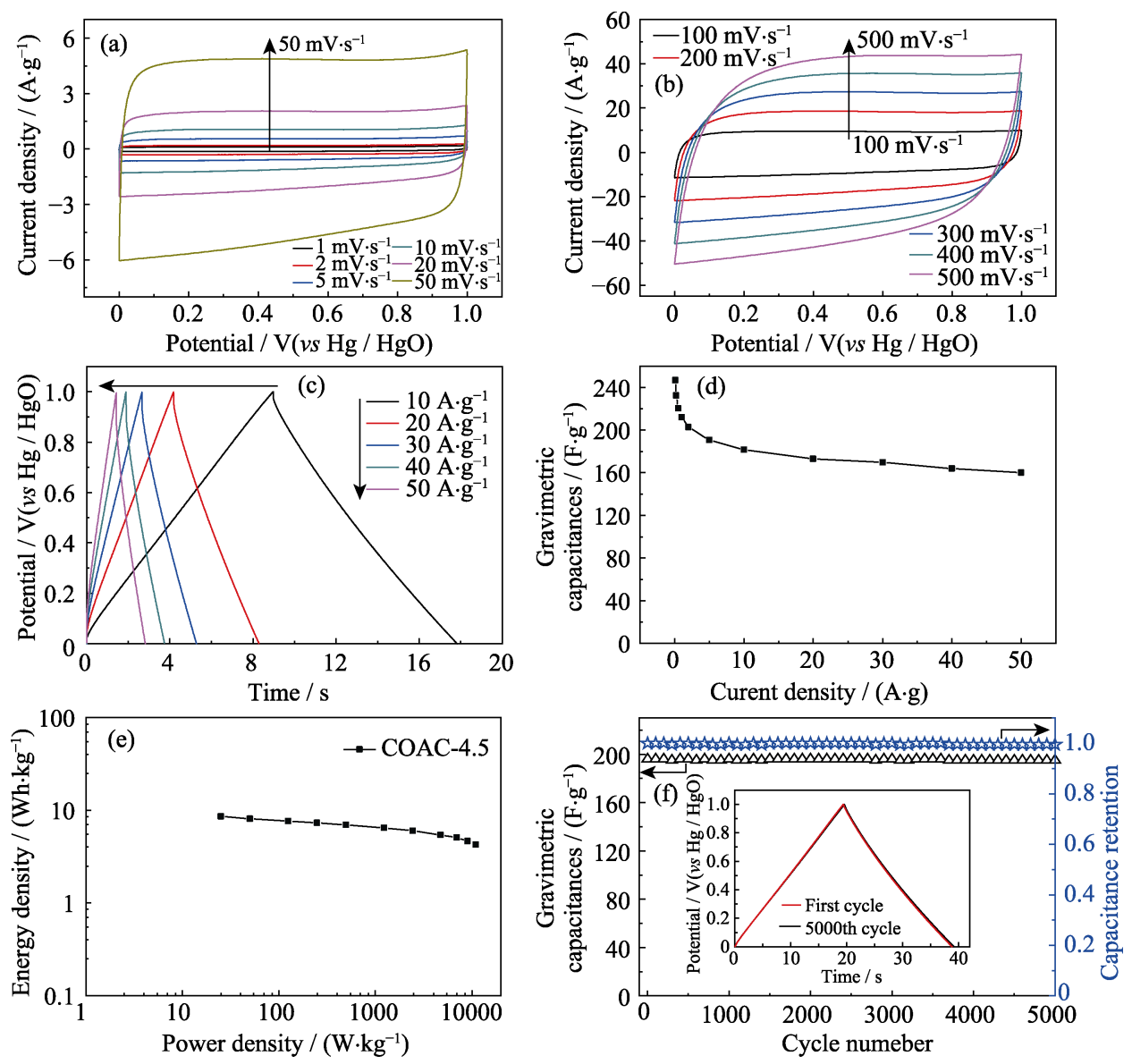

图 S1 样品 COAC-4.5 在 $6 \mathrm{~mol} / \mathrm{L} \mathrm{KOH}$ 两电极体系的电化学性能: (a)在扫描速率 1 50 mV/s 下的循环伏安曲线; (b)在扫描速率 100 500 mV/s 下的循环伏安曲线; (c)在电流密度 10 50 A/g 下的恒电流充放电曲线; (d) 比电容与 电流密度函数关系曲线; (e)能量密度与功率密度关系曲线; (f) 在电流密度 $5 \mathrm{~A} / \mathrm{g}$ 下循环 5000 次的长循环曲线 (插图为第 1 次与第 5000 次循环恒电流充放电曲线)

Fig. S1 Electrochemical performance of COAC-4.5 measured in two electrode system with $6 \mathrm{~mol} / \mathrm{L} \mathrm{KOH}$ electrolyte:

(a), (b) CV curves at different scan rates; (c) Galvanostatic charge-discharge curves at different current densities;

(d) Specific capacitances for a single electrode at different current densities; (e) Ragone plot of the symmetrical system; (f) Cycling stability at a current density of $5 \mathrm{~A} / \mathrm{g}$ and inset is the charge-discharge curves of first cycle and 5000th cycle 

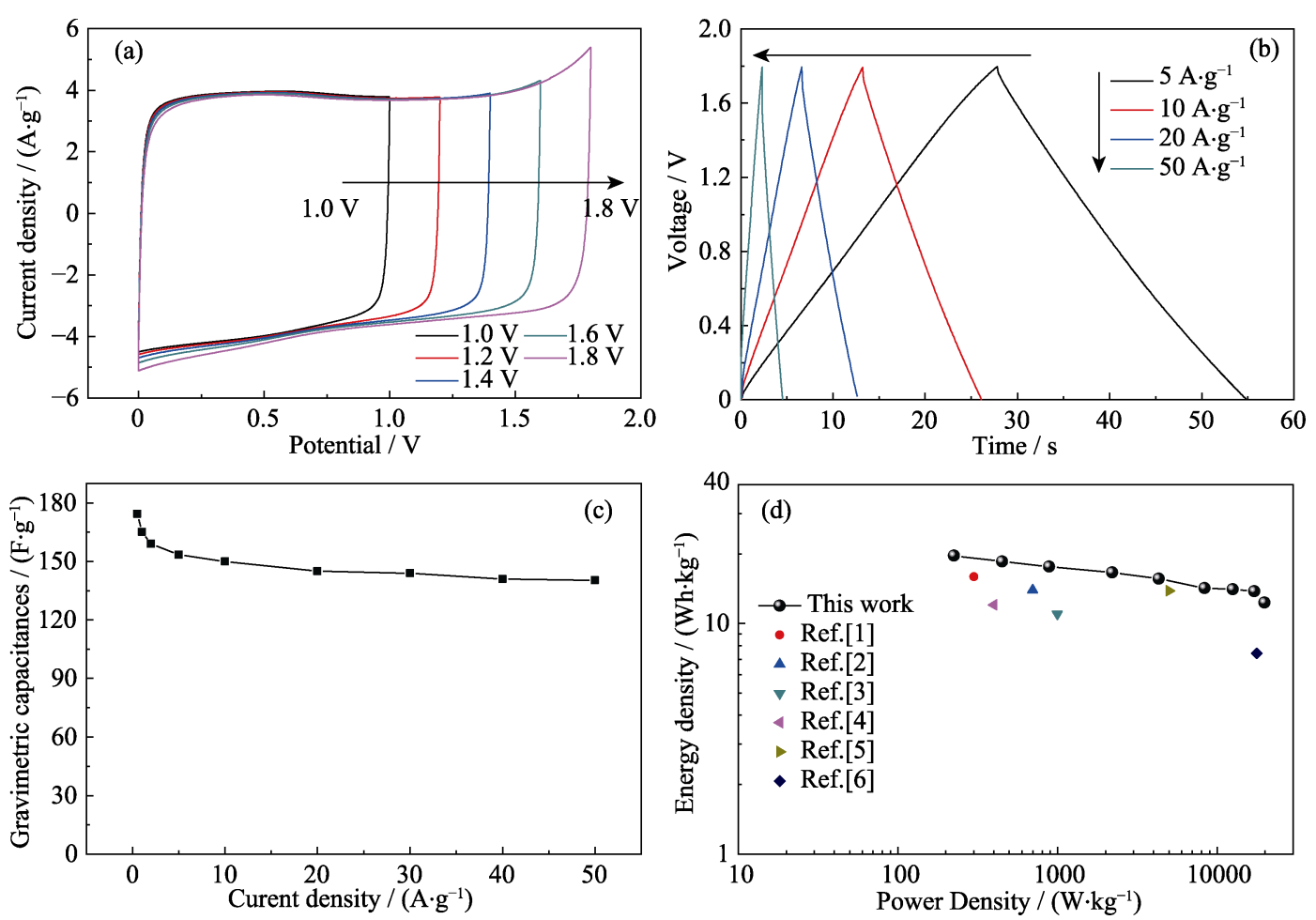

图 S2 样品 COAC-4.5 在 $1 \mathrm{~mol} / \mathrm{L} \mathrm{Na}_{2} \mathrm{SO}_{4}$ 两电极体系的电化学性能: (a)在不同电势窗口、 扫描速率 $50 \mathrm{mV} / \mathrm{s}$ 下的循环伏安曲线; (b)在电流密度 $5-50 \mathrm{~A} / \mathrm{g}$ 下的恒电流充放电曲线;

(c)比电容与电流密度函数关系曲线; (d) 能量密度与功率密度关系曲线

Fig. S2 Electrochemical performance of COAC-4.5 measured in two electrode system with $1 \mathrm{~mol} / \mathrm{L} \mathrm{Na}_{2} \mathrm{SO}_{4}$ electrolyte:

(a) CV curves of the cell operated in different voltage windows at a scan rate of $50 \mathrm{mV} / \mathrm{s}$; (b) Galvanostatic charge/discharge curves of the cell at various current densities; (c) Specific capacitances for a single electrode at different current densities;

(d) Ragone plot of COAC-4.5 and other carbon-based symmetrical supercapacitors

表 S2 XPS 分析样品 COAC-n 的 C, O 和 N 元素含量

Table S2 C, O and $N$ contents of COAC-n samples from XPS analysis

\begin{tabular}{ccccccc}
\hline Samples & $\mathrm{N} / \mathrm{at} \%$ & $\mathrm{C} / \mathrm{at} \%$ & $\mathrm{O} / \mathrm{at} \%$ & $\mathrm{O}-\mathrm{I} / \mathrm{at} \%$ & $\mathrm{O}-\mathrm{II} / \mathrm{at} \%$ & $\mathrm{O}-\mathrm{III} / \mathrm{at} \%$ \\
\hline COAC-3.5 & - & 86.65 & 13.35 & 5.85 & 4.58 & 2.92 \\
COAC-4.0 & 1.42 & 81.51 & 17.07 & 7.87 & 5.78 & 3.42 \\
COAC-4.5 & 1.34 & 86.12 & 12.54 & 4.04 & 3.77 & 4.73 \\
COAC-5.0 & 1.37 & 82.79 & 15.84 & 7.78 & 5.24 & 2.82 \\
\hline
\end{tabular}

\section{参考文献:}

[1] WANG Q, YAN J, WANG Y B, et al. Three-dimensional flower-like and hierarchical porous carbon materials as high-rate performance electrodes for supercapacitors. Carbon, 2014, 67(2): 119-127.

[2] SUN G L, LI B, RAN J B, et al. Three-dimensional hierarchical porous carbon/graphene composites derived from graphene oxide-chitosan hydrogels for high performance supercapacitors. Electrochimica Acta, 2015, 171: 13-22.

[3] LONG C L, CHEN X, JIANG L L, et al. Porous layer-stacking carbon derived from in-built template in biomass for high volumetric performance supercapacitors. Nano Energy, 2015, 12: 141-151.

[4] BELlO A, BARZEGAR F, MOMODU D, et al. Symmetric supercapacitors based on porous 3D interconnected carbon framework. Electrochimica Acta, 2015, 151: 386-392.

[5] BELLO A, MANYALA N, BARZEGAR F, et al. Renewable pine cone biomass derived carbon materials for supercapacitor application. $R S C$ Advances, 2016, 6(3): 1800-1809.

[6] SHAN D, YANG J, LIU W, et al. Biomass-derived three-dimensional honeycomb-like hierarchical structured carbon for ultrahigh energy density asymmetric supercapacitors. Journal of Materials Chemistry A, 2016, 4(35): 13589-13602. 